\title{
A Research on Characteristics and Development Trend of Sports Commu- nication in the Era of Big Data
}

\author{
Ming Shi ${ }^{*}$
}

Department of Leisure and Social Sports, Harbin Institute of Physical Education, Harbin 150090, Heilongjiang, China

\begin{abstract}
This paper discusses the current situation of sports communication in the big data era and the relationship between the big data and the media. Then describe characteristics of the big data era sports communication through three aspects : the channel platform, audience boundaries and present content, and predict the future development of the big data era sports communication through four aspects: enhance information analysis, upgrade their own media literacy, build cross media integrated communication mode and the government provides the system guarantee. Conclusion: large data has been used in every industry of sports, for sports communication has great role in promoting, it promotes the development of undertakings of physical culture and sports. However, at present, China is still in the initial stage, it needs seize the opportunity to pull the sports communication into an all-round way to the information in the future development.
\end{abstract}

Keywords: Big data, media literacy, sports communication, system guarantee.

\section{INTRODUCTION}

Big data [1] as a wave swept almost all the world-class industry, especially in the marketing industry and the information industry, and the preliminary operation of the big data is absolutely necessary, meanwhile, the big data successful cases can be founded everywhere. The whole world exclaimed: big data era have already arrived (see Fig. 1).

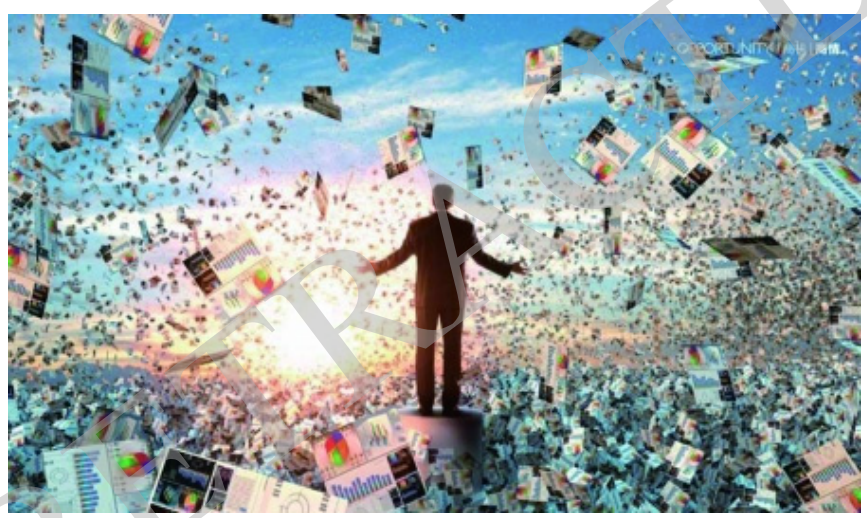

Fig. (1). Big data is used in almost every industry.

Thus, many experts and scholars said that the year of 2013 was the first year of big data, and the public tend to agree with this viewpoint. In fact, the big data has profoundly affected all areas of human society, which bringing the information field to the new era of revolution. Sports field has always been the field of the highest frequency of information utilization, the "high" and the "wide" of the information use determines the level of sports performance [2]. The most sensitive to the information dissemination of

*Address correspondence to this author at the Department of Leisure and Social Sports, Harbin Institute of Physical Education, Harbin 150090, Heilongjiang, China; E-mail: 2608201199@qq.com the media for new things has the greatest ability to accept and communication, it put big data concept throughout all areas. Facing towards the 2016 Olympic Games in Rio de Janeiro, big data can be a new angle of view for the development of the sports media? This paper intends to clarify the concept of "big data", which will pave the way for the development of media communication in big data era, so as to discuss the sports communication in the background of big data.

\section{ANALYSIS ON THE CURRENT SITUATION OF THE DEVELOPMENT OF SPORTS COMMUNICA- TION IN THE BIG DATA ERA}

Professor Huang Shengmin [3], a famous communication expert at the Communication University of China, who wrote a book named how about the TV in the big data era, in this book he think that through changing the human data processing methods, ideas and abilities big data affect all aspects of human social life, in the subversion of the old system, and also provides the possibility of reconstruction of a new ecosystem. It can be said that the development of big data and media is in a mutually beneficial relationship.

\subsection{Big Data Can Promote the Integration of Traditional Media and New Media in Sports Communication}

For many years, the sports news communication has been the main media products, and it is the main way to spread the Olympic spirit and culture. Sports journalism experts Haoqin professor pointed out: the early stage of the Olympic Games is limited by the time, which only depend on speaking, meeting, newspapers and periodicals, etc. In the first session of the Olympic Games, Coubertin can rely on the means of the speech, the meeting; what's more, it also can depend on the communications magazine as well as the Greek messenger. 
In 1896, the first Modern Olympic Games held in Athens, at that time, the International Olympic Committee (IOC) [4] publicized the "communication", which undertake the important mission of the Olympic information and the dissemination of Olympic culture, while the Athens' "messenger" is the main newspaper that published the first Olympic Games. The Olympic Games has brought about a fever of sports communication, which brings the development of the sports newspapers, magazines, and it also brings challenges. But it is impossible to find the newspaper information in that time. This transformation has been formed in the computer age, and it can be used for the information value. Now the era of big data with cloud computing on the basis of a large number of data stored, then use the electronic platform analysis, prediction of news events, to make up for the disadvantaged media.

\subsection{Big Data Can Forecast the Media Market}

It is well known that big data is a kind of forecast method [5] based on relevant data. Russia try to increase efforts to promote mass sports is the most important part of a healthy lifestyle. In order to achieve the development of the Russian federal government for the development of mass sports, firstly the government put the publicize work as a breakthrough, then study on the interests, needs and motivations of different groups of people, so as to change the whole Russian people's conception and attitude to the physical exercise.

\subsection{Big Data Can Forecast the Media Market}

The development of all media in 2013 cannot ignore the impact of big data; and the media has increased the big data to a strategic level, In other words, in order to win the initiative of development, the strategic level need to be comprehensive and long-term deployment and robust. The reason why big data can rein the media is because the most use of the big data technology, and there is no doubt that it is the basic foundation. We can use the big data to focus on the future trend of the forecast, and the media also strive to achieve a significant increase in the value of large data information.

The house of cards is sweep around the world, which was praised as the first count out of the TV drama by the media in the big data era, and the overseas media treat it as the "data revolution" representative. "House of cards" is a United States political drama, which made by the video site Netflix [6], at the same time, this site analyze 30000000 users in the TV series consumption habits database and investigate 10000000 US dollars technology to create the drama. In this database, it has 30000000 users of viewing options, 4000000 reviews, and 3000000 subject searches; of course it is a veritable big data. The company use data analysis and forecasting technology successfully develop strategies, and shepherds "the house of cards" became popular, which confirms the power of big data and the influence of big data can be seen in it.

\section{THE CHARACTERISTICS OF SPORTS COMMU- NICATION IN THE ERA OF BIG DATA}

News communication is the first industry which has a real feeling of the Internet industry, and there is no doubt that the advent of the era of big data has a great influence to the news industry [7]. Based on the social environment of the era of big data and from the perspective of the overall trend of the development of the information and communication technology, Sports media is being spread from the paper media sports communication mode to the digital sports transmission mode and the cloud digital sports communication model, (see Fig. 2) from the single mode of physical education to the interactive and social sports communication mode, from the fixed mode of transmission to the mobile and intelligent mobile communication mode. Its specific characteristics are reflected in the following 3 aspects:

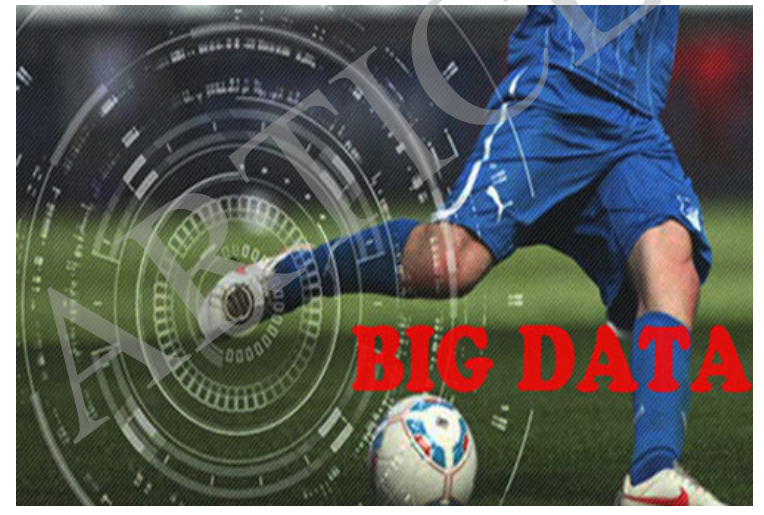

Fig. (2). Big data has been used in the media of sports new communication for years.

\subsection{Large Data Sports Communication Platform for the Selection is Diversity}

In the big data era, based on the diversity of communication content and mode, the market will provide more technical platform and channel for the dissemination of sports information. At this point, the emergence of multi sports information dissemination platform and channel, which reduces the dependence on the traditional TV media, even in part. Meanwhile, the new platform has a positive meaning for improving the quality of sports communication, including the demand for the content of the network. Sports information dissemination channel and the monopoly platform by telecommunications and Internet companies were be break, and appeared in addition to the radio and television system, which can achieve production, broadcast, service platform system. From the content perspective, the big data era of radio and television sports continue to promote the construction and transmission of high-definition interactive content of the main business, we can get more development and rich customer experience [7]. For telecommunications companies, this is to accelerate the establishment of sports communication content resources, and quickly get the audience to provide an opportunity. Based on the competition pattern of the era of big data of different sports communication platform and channel can greatly stimulate the "production" more high-quality sports information resources, and promote the healthy development of sports media and sports itself. 


\subsection{The Ambiguity of Audience Boundaries of Big Data Sports Communicators}

In the era of big data, based on the transformation of the mode of transmission, the source and structure of sports communication has also changed subtly. Due to the diversity of data sources, the distribution channel of information is no longer limited to the authority and traditional media organizations, the sports media and the audience has gradually lost the traditional sense of dividing line. In network technology and media technology rapid development today, accept the audience of sports information and release information of sports communicators function gradually fusion, makes the general audience with the traditional sports disseminators of information acquisition, analysis, release function. Subsequently, the production of sports information, publishing process also produces a revolutionary change. However, when the different audience into sports reporter, the different to traditional sports reporter's editorial ideas and practices, they may pay more attention to the sports information in the course of information collection, "the original ecology" [8] and according to their own interest in sports personalized release. When after the establishment of the sports communication, sports information dissemination by the relationship changed, both sides is no longer exists obvious boundaries, personalized and niche sports information in mass communication system, making sports information people receive more personalized and diversified.

\subsection{Fragmentation of Big Data Sports Dissemination}

Based on the "public", the "micro", the "fast" and "short" of the news, the "short" and "short" of the big data era, the content of the sports dissemination is characterized by "fragmentation". Fragmented text has attracted the audience to use intermittent and fragmented time to focus on sports information. But this property also determines the it is a kind of "unfinished version" [9]. The information element is isolated and background information is missing, so that the audience can understand the information context. As in the micro Bo, the brief event results reported, sports commentator's opinion, the interaction between athletes and users, as well as a variety of hot discussion of public opinion is a more common form. These contents are brief and scattered, which have the characteristics of fragmentation and personalization, but it emphasizes the communication between people and people, and it is the collection of ideas and thoughts. Due to include adding from the media, new media, information release, lowering the threshold, many information release due to personal color strong and often difficult to achieve the professional requirements, showing a valuable "grassroots" [10], but also false news of ills. In the era of big data, due to the pursuit of news media release rate and attract the attention of the audience and ignore the accuracy of news and professional, for professional requirements higher sports news reports, fragmentation of information transmitted by a variety of channels, often on the content of quality and authenticity unable to control. In the era of big data, although more and more people begin to pay attention to sports events and sports information through various channels to understand, but more and more audience to receive information is incomplete, no accurate or even wrong.
4. THE FUTURE DEVELOPMENT DIRECTION OF SPORTS COMMUNICATION IN BIG DATA ERA

\subsection{Sports Communication Group Enhanced Informa- tion Analysis and Interpretation Ability, Effective Pres- entation of Data}

As mentioned earlier, the big data era information disseminators and the audience the blurring of lines, but specific communicative contexts and events, disseminators and the audience points belong to different subjects [11], with different duty and value orientation. Big data era is a mass production, sharing and application data era. Based on this background, in recent years, the sports dissemination of information also showed a massive growth trend, the disorder of the sports information and clutter makes people in the choice of a suitable for their own information, increased time and material cost. Because of the sports communication can't effectively provide valuable sports information, and the audience of sports information processing capacity, so that people get the actual efficiency of sports information. As professor Victor said: "the real value of the big data is like the tip of an iceberg floating in the ocean, the first sight of the iceberg, and most of them are hidden beneath the surface."[12] So for the dissemination of sports, in the era of big data, enhance information analysis and interpretation ability, to explore in depth behind the sports data information and its real meaning, be the most exciting and the most loved by the audience, "rich is energy" sports information [13] presented in front of the audience, is the big data era sports dissemination of survival and direction. Sports reporters, editors should not only be proficient in the use of text, images, but also need to know how to gather, integrate, mining and analysis of data

\subsection{Sports Dissemination Benefit Groups Improve Their Media Literacy, Rational Choice of Data}

In the era of big data, the audience with media sports information access to increased autonomy, but due to the differences in social background, educational degree and so on, a class of people can effectively using data of sports communication technology to understand the dynamic PE [14], collect scientific fitness method, positive sports commentary, and benefit from it; and the other people use just browsing sports information, lively scene, a simple sports communication for pure entertainment, emotional catharsis. Effectively using the era of big data of sports information communication can mobilize national fitness, promote sports culture, popularizing the knowledge of sports science, but due to differences in the audiences' media literacy, resulting in sports information using improper, sports information distortion and other issues. To solve these problems requires not only new media strengthen self-discipline, to enhance the dissemination of data processing ability, purify the propagation environment, provide healthy sports information data, also need the audience to improve their media literacy, learn to discern information authenticity, using its rational judgment to select the useful information and sports data, realize benign dissemination of sports information. The era of big data change not only the audience access to information on the attitudes and behavior of, more is on traditional news 
gathering concept and process the solution and reconstruction, is the challenge and innovation of theory of classic journalism, communication science. Physical structure of the source, the production process of news and media terminal presentation by the profound impact of sports communication way change, thus enhancing the audience's media literacy and information judgment ability is imminent.

\subsection{Sports Media to Create Cross Media Integrated Communication Mode, Efficient Integration of Data}

In the era of big data, targets for the development of sports media communication mode is on cloud computing, formed to sports information platform for driving engine, database application kernel services, new media, cross media integrated marketing communication mode of distribution channels and platforms. Because through the cloud computing, can be abundant basic information for storage, sharing and mining, convenient and effective will these large, highspeed, a number of changes to the terminal data storage, cloud based calculation technique can at any time analysis and calling. At the same time, in the existing graphics and text, audio and video communications, the advantages of a variety of media should be set to create a cross media communication mode, so that the data has a visual effect. "Forbes" magazine for more than 100 years to the winning of the Nobel Prize a visual display [15], makes the audience from the complex data to obtain the most important information, and achieved a better communication effect.

4.4. Government and Related Organizations to Provide Policy and System Security, to Fulfill The Functions of Supervision and Service

The government and relevant departments should be based on the complexity of the era of big data dissemination of sports information data is the introduction of relevant policies, laws, regulations, rules and other, sports communication data effective integration, "in order to protect the healthy development of sports communication at the same time, in the government's strategic planning level, try to establish the sports media data warehouse, in the field of sports communication implementation of the concept of the minimum data set, collected in individual sports media data, in guarantee sports information and culture security under the premise of effective management of sports data resources, to realize the benign interaction between the dissemination of sports information resources and sharing of the era of big data. At the same time, the government and related sports organizations should carry out the responsibility in the sports communication data integration and sports information service. For example, Shanghai is building public sports information platform "for ordinary citizens receiving effective and useful sports information daily fitness provides a great convenience, through the platform of the public can easily obtain fitness guidance information, venue information, booking information, and in line with their own exercise prescription information and data, through the mobile app and sports social information service, make exercise into people's daily work and life, so as to realize the big data era of sports information and data integration, supply and utilization.

\section{CONCLUSION}

China is a developing country, and our country is rapidly heading for information and networking, it seems that we hand over a god report card in the process. At present, big data is likely to become a competitive tool to the developed countries in the next round of global competition, while the developing countries are still in a passive state of dependence. In order to catch up with such a big data transformation, our country should try to open the data and methods to fully tap the data, and no longer pursue causality. At the same time, we should recognize the confounding, explore the relationship and change the thinking method. By means of big data innovation approach to embrace the big data era, in this way we can seize the opportunity to create history.

\section{CONFLICT OF INTEREST}

The author confirms that this article content has no conflict of interest.

\section{ACKNOWLEDGEMENTS}

\section{Declared none.}

\section{REFERENCES}

[1] F. Provost and T. Fawcett, Authors' Response to Gong's, "Comment on data science and its relationship to big data and datadriven decision making", Big Data, vol. 2, no. 1, pp. 1-1, 2014.

[2] X. Wu, X. Zhu, G.Q. Wu and W. Ding, "Data mining with big data", IEEE Transactions on Knowledge and Data Engineering, vol. 26, no. 1, pp. 97-107, 2014.

[3] D. Lazer, R. Kennedy, G. King and A. Vespignani, "The parable of Google Flu: traps in big data analysis”, Science, vol. 343, no. 6176, pp. 1203-1205, 2014.

[4] D. Tuckett, R. Nyman, P. Ormerod, and R. Smith, Big Data and Economic Forecasting: A Top-Down Approach Using Directed Algorithmic Text Analysis, ECB Workshop on Big Data for Forecasting and Statistics, Frankfurt, 7-8 April 2014.

[5] J. Lane, V. Stodden, S. Bender and H. Nissenbaum, Privacy, Big Data, and the Public Good: Frameworks for Engagement, Cambridge University Press, UK, 2014.

[6] H.R. Varian, "Big data: New tricks for econometrics", The Journal of Economic Perspectives, vol. 28, no. 2, pp. 3-27, 2014.

[7] S. Menon, Biodiversity Informatics-Big Data for Biodiversity Conservation and Ecological Forecasting, Grand Valley State University, 2014.

[8] G. George, M.R. Haas and A. Pentland, "Big data and management", Academy of Management Journal, vol. 57, no. 2, pp. 321326, 2014.

[9] C. Von Sikorski and T. Schierl, "Effects of news frames on recipients' information processing in disability sports communications", Journal of Media Psychology, vol. 24, no. 3, pp. 113-123, 2015.

[10] R. White and M. Cheung, "Communication of fantasy sports: a comparative study of user-generated content by professional and amateur writers", IEEE Transactions on Professional Communication, vol. 58, no. 2, pp. 192-207, 2015.

[11] B. McCarthy, "A sports journalism of their own an investigation into the motivations, behaviours, and media attitudes of fan sports bloggers", Communication \& Sport, vol. 2, no. 1, pp. 65-79, 2014.

[12] T.B. Cornwell, Sponsorship in Marketing: Effective Communication Through Sports, Arts and Events. Routledge, UK, 2014.

[13] J.S. Lim, Y.C. Hwang, S. Kim, and F.A. Biocca, "How social media engagement leads to sports channel loyalty: Mediating roles of social presence and channel commitment", Computers in Human Behavior, vol. 46, pp. 158-167, 2015. 
[14] Z. Onağ, and M. Tepeci, "Team effectiveness in sport teams: the effects of team cohesion, intra team communication and team norms on team member satisfaction and intent to remain", Procedia-Social and Behavioral Sciences, vol. 150, pp. 420-428, 2014.
[15] A. Godoy-Pressland, "The weekend as a male entity: how Sunday newspaper sports reporting centres around male activities, interests and language (2008 and 2009)", Leisure Studies, vol. 33, no. 2, pp. 148-163, 2014.

Received: June 10,2015

Revised: July 29, 2015

Accepted: August 15, 2015

(C) Ming Shi; Licensee Bentham Open.

This is an open access article licensed under the terms of the (https://creativecommons.org/licenses/by/4.0/legalcode), which permits unrestricted, noncommercial use, distribution and reproduction in any medium, provided the work is properly cited. 